\title{
Non-mass lesions on screening breast ultrasound
}

\author{
Jihyun Lee ${ }^{1}$, Jin Hwa Lee', Seonmi Baik², Eun Cho ${ }^{1,3}$, Dong won Kim¹, Hee Jin Kwon", \\ Eun-Kyung $\mathrm{Kim}^{3}$
}

${ }^{1}$ Department of Radiology, Dong-A University Medical Center, Dong-A University College of Medicine, Busan, ${ }^{2}$ Department of Radiology, Haeundae Sharing and Happiness Hospital, Busan, ${ }^{3}$ Department of Radiology and Research Institute of Radiological Science, Severance Hospital, Yonsei University College of Medicine, Seoul, Republic of Korea

\begin{abstract}
Aim: The purpose of this study was to determine the significance of a non-mass lesion (NML) which is recognized during screening breast ultrasound (US). Materials and methods: We included patients with a NML on screening breast US and no suspicious finding on mammography between March 2008 and June 2012. The final diagnoses were based on pathology results and a clinical or sonographic follow-up for more than 12 months. We calculated the incidence, likelihood of malignancy, and positive predictive value (PPV) of biopsy with a review of imaging and histopathological findings. Results: A total of 17868 screening breast US were performed in 8856 asymptomatic patients. Ninety-five NMLs were detected in 88 patients (1.0\%). Among the 93 lesions that were followed or confirmed histopathologically, $2(2.2 \%)$ were malignant, $89(95.6 \%)$ were benign, and $2(2.2 \%)$ were high risk lesions. The likelihood of malignancy in a NML on screening breast US was $2.2 \%$ and the PPV of biopsy was $6.3 \%$ ( 2 of 32). Conclusion: The likelihood of malignancy for a NML on screening breast US was greater than $2 \%$. It could be classified as a BI-RADS category $4 \mathrm{a}$ lesion and tissue diagnosis is warranted. This provides the potential management guidelines for a NML in screening patients and further prospective studies in a large, multicenter screening population are required.
\end{abstract}

Keywords: breast, ultrasound, cancer screening, non-mass lesion

\section{Introduction}

With the use of high-resolution ultrasound (US) equipment and the recent introduction of supplementary screening breast US in a population with dense breasts and an elevated risk of breast cancer, the hypoechoic nonmass lesions (NML) that do not meet the criteria of a mass defined by the American College of Radiology Breast Imaging Reporting And Data System (ACR BI-RADS) lexicon [1] are sometimes encountered [2-4]. It has been reported that these lesions on US can reflect a wide spectrum of pathologic changes such as fibrocystic change, fibrosis, mastitis, ductal carcinoma in situ (DCIS), invasive

Received 28.04.2016 Accepted 13.07.2016

Med Ultrason

2016, Vol. 18, No 4, 446-451

Corresponding author: Jin Hwa Lee, MD,

Department of Radiology,

Dong-A University Medical Center,

Dong-A University College of Medicine,

26, Daesingongwon-ro, Seo-gu,

Busan 49201, Republic of Korea

Phone: 82-51-240-5368, Fax: 82-51-253-4931

E-mail: jhrad@dau.ac.kr lobular carcinoma (ILC), or invasive ductal carcinoma (IDC) [3-12]. However, these NMLs are not included in the BI-RADS lexicon for breast US [1].

The Japanese Association of Breast and Thyroid Sonology systematically organized and classified NMLs, first time in 2004 [2], but only a few studies have reported the imaging features and histologic characteristics of NMLs $[3,6,13]$. Moreover, no report has been published regarding the incidence, BI-RADS categorization, and the guidelines for management of NMLs on screening breast US. Therefore, the purpose of this study was to investigate the incidence, histologic characteristics and US features of NMLs which were recognized during screening breast US and to determine the positive predictive value (PPV) of biopsy and the likelihood of malignancy of these features.

\section{Materials and methods}

\section{Patients selection and inclusion criteria}

Institutional review board approval was obtained for this retrospective study, and the informed consent was waived. A total of 17868 bilateral whole-breast 


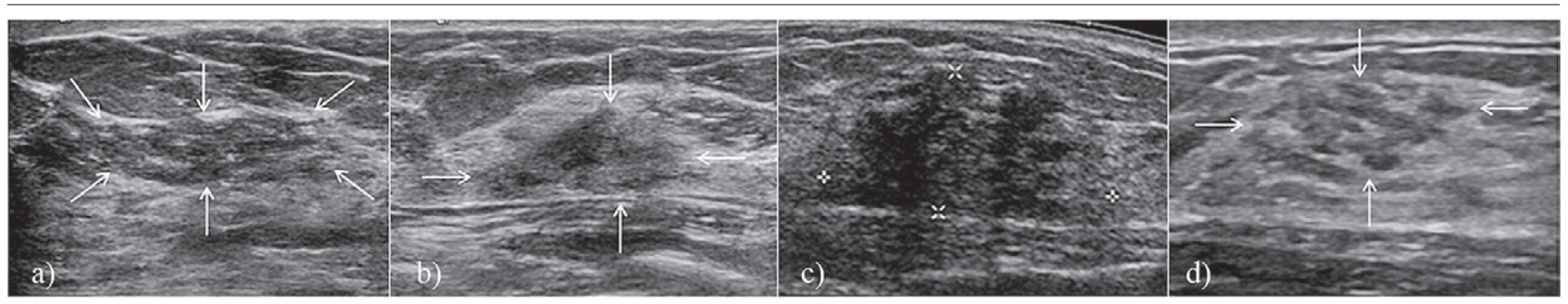

Fig 1. Ultrasonic classification of non-mass lesions. Sonograms show indistinct (a,b), geographic (c), and mottled (d, Courtesy of Kim SJ, Busan, Republic of Korea) patterns.

US screening were performed in 8856 asymptomatic women at our institution between March 2008 and June 2012. Indications for screening breast US were as follows: asymptomatic patients with dense breast tissue on mammography, postoperative screening after surgery for breast cancer, and screening which is requested by patients themselves or physicians. Among these examinations, 513 patients with NMLs were retrospectively identified through a search of our radiology database with a prospectively recorded NML. A non-mass lesion was defined as a hypoechoic area which does not conform to the definition of a "mass", which is defined as a spaceoccupying lesion seen in two different planes and it has different character from that of the surrounding parenchyma or the same area in the contralateral breast $[2,3]$. It may be analogous to a focal asymmetry on mammography. Lesions showing the patterns of duct changes were excluded from this study. Among them, we excluded 388 patients with a past history of biopsy or operation at the area of a NML and 37 patients with mammographic suspicious features of malignancy and finally, a total of 88 patients with 95 lesions were included in this study. Seven patients had two lesions. The patients ranged in age from 28 to 68 years, with a mean age of 48.2 years.

\section{Image analysis}

Bilateral whole-breast US examinations were performed by one of two breast imaging radiologists with 12 and 9 years of experience in breast US, respectively. High-resolution units with $7-13-\mathrm{MHz}$ or $5-12-\mathrm{MHz}$ linear-array transducers (Sonoline Antares, Siemens, Issaquah, Wash; HDI-5000, Philips-ATL, Bothell, WA) were used. Grayscale US was first performed to evaluate the sonographic characteristics of NMLs. A minimum of 2 orthogonal grayscale images of each lesion were obtained. Extended field-of-view (FOV) technology was used when the lesion size exceeded the FOV of the probe. After that, power Doppler US was performed.

The management of NMLs was depended on operator experience and clinical judgement. When a NML was detected, short-term follow-up after 6 months or tissue diagnosis was recommended. The indications for tissue diagnosis were as follows: NMLs with more hypoechoic patterns, lesions with posterior shadowing, new or increasing lesions compared with prior US images if available, lesions in high-risk women, patients' preference or clinical request to perform a biopsy. In cases that these criteria were not met, short-term follow up after 6 months was recommended.

The retrospective review for the classification of US findings of NMLs was based on consensus between two dedicated breast radiologists (J.H.L. and S.M.B) and the readers were blinded to the final diagnosis. The lesions were classified into indistinct, geographic, and mottled groups by US patterns according to the antecedent studies about NMLs on breast US [2,3]. An indistinct pattern was defined as a relatively uniform hypoechoic area whose margins were not clearly defined (fig 1a-b). A geographic pattern was defined as a confluent hypoechoic area with a cobblestone appearance including an aggregation of small, island-like low echoic areas (fig 1c). A mottled pattern was defined as a number of small, island-like low echoic areas in the mammary parenchyma (fig 1d). US distribution classified NMLs into focal and regional groups. A focal distribution was defined as a lesion which was scanned within the FOV of the linear probe and a regional distribution was defined as a lesion which exceeded the FOV and needed the use of extended FOV technology. The internal vascularity of the lesions on power Doppler images was classified into absent and present groups.

\section{Establishment of the final diagnosis}

The final diagnoses were based on pathology results and a clinical or sonographic follow-up for more than 12 months. Pathologic results, whether obtained by US-guided core needle or excisional biopsy, were considered definitive. US-guided biopsy was performed using a 14-gauge semi-automated core biopsy needle (Stericut coaxial; TSK Laboratory, Tochigi, Japan) or a handheld vacuum-assisted device with an 8-gauge probe (Mammotome; Devicor Medical Products; Cincinnati, OH, USA). In cases of a high risk lesion diagnosed by core needle biopsy, surgical excision or handheld vacuum-assisted biopsy were recommended. Lesions placed into imaging surveillance were considered benign if they were stable for at least 12 months. 
We calculated the incidence, PPV of biopsy, and likelihood of malignancy in the NMLs on screening breast US.

\section{Results}

The incidence of the NML on screening breast US was one percent (95 lesions in 88 patients from a total of 8856 patients) at a single institution. Figure 2 shows the follow-up and pathology results. The mean follow-up interval was 33 months, with a range of $0 \sim 84$ months and two patients with two lesions were lost to follow-up. All sixty-one lesions that were followed did not change or decrease in size during follow-up. US -guided core nee-

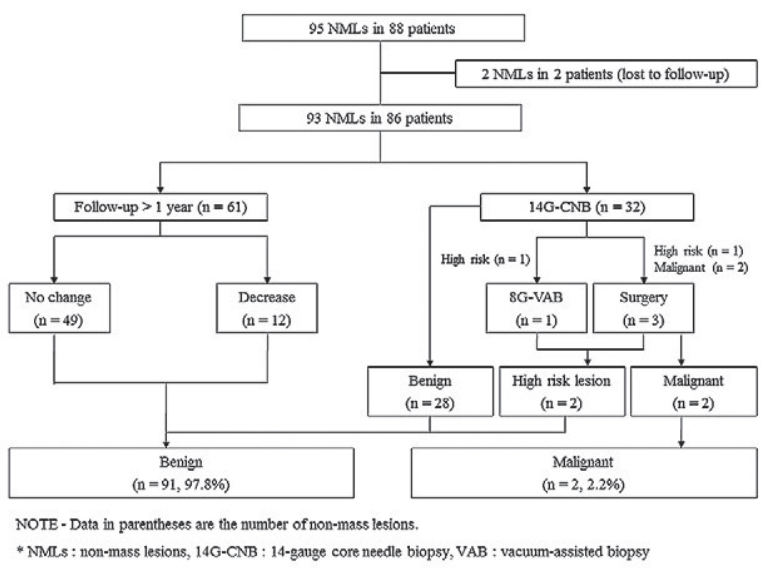

Fig 2. Algorithm that shows follow-up and pathology results.

Table I. Histological characteristics of the pathologically confirmed non-mass lesions

\begin{tabular}{ll}
\hline Histological Characteristics $(\mathbf{n}=\mathbf{3 2})$ & No. \\
\hline Benign $(\mathrm{n}=30)$ & 8 \\
Fibrocystic change & 3 \\
Adenosis & 3 \\
Fibrosis & 3 \\
Stromal hyalinization & 2 \\
Ductal hyperplasia & 2 \\
Diabetic fibrous mastopathy & 2 \\
Sclerosing adenosis & 2 \\
Stromal fibrosis & 1 \\
Chronic periductal inflammation & 1 \\
Lactation foci & 1 \\
Pseudoangiomatous stromal hyperplasia & 1 \\
Atypical ductal hyperplasia & 1 \\
Intraductal papilloma & \\
Malignant (n = 2) & 1 \\
Ductal carcinoma in situ & 1 \\
Intraductal papillary carcinoma & \\
\hline
\end{tabular}

Note - Data are the number of lesions. dle biopsy was performed in 32 lesions and among them, 28 were benign, 2 were high risk lesions and 2 were malignant. In cases of high risk lesions, further treatment including a vacuum-assisted biopsy and excision was performed and they were finally confirmed as atypical ductal hyperplasia and intraductal papilloma, respectively.

Therefore, among the 93 lesions which were confirmed by imaging follow-up or pathology, 2 were malignant and the likelihood of malignancy was $2.2 \%$ and the PPV of biopsy was $6.3 \%$. Table I demonstrates the his-

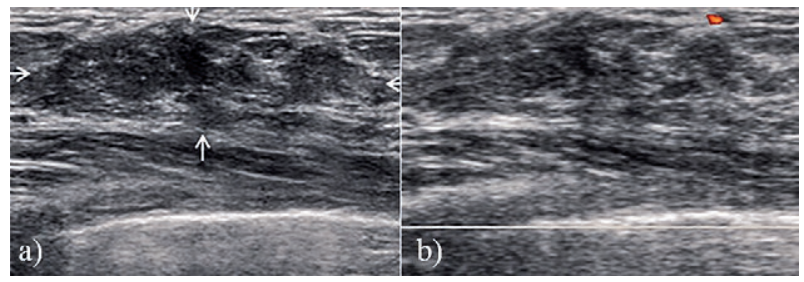

Fig 3. A 51-year old woman presented for screening examination. Grayscale US showed a non-mass lesion with an indistinct pattern and a focal distribution at the 10 o'clock position in the right breast (a). On power Doppler image, internal vascularity was not noted in this lesion (b). Fibrocystic change with marked fibrosis was confirmed by a US-guided core needle biopsy.

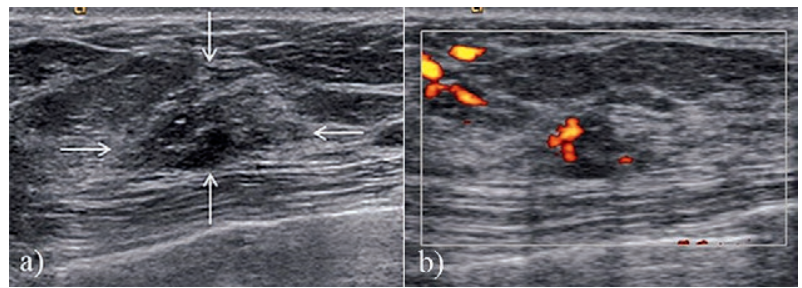

Fig 4. A 52-year-old asymptomatic woman, who had undergone a mastectomy for contralateral breast cancer 3 years ago. Postoperative screening US demonstrated a non-mass lesion showing an indistinct pattern and focal distribution at the 2 o'clock position in the right breast (a). Internal vascularity was observed on a power Doppler image (b). A ductal carcinoma in situ was confirmed by a US-guided core needle biopsy and a subsequent mastectomy was performed.

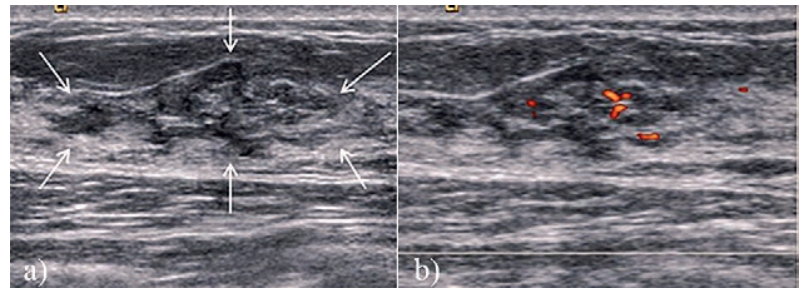

Fig 5. A 55-year-old asymptomatic woman presented for screening examination. Screening US demonstrated a non-mass lesion showing an indistinct pattern and a focal distribution at the 12 o'clock position in the left breast (a). Internal vascularity was observed on a power Doppler image (b). An intraductal papillary carcinoma was confirmed by a US-guided core needle biopsy and a subsequent breast conserving surgery was performed. 
Table II. US features of non-mass lesions

\begin{tabular}{ll}
\hline Non-mass lesions $(\mathbf{n}=\mathbf{9 3})$ & Number $(\%)$ \\
\hline US pattern $(\mathrm{n}=93)$ & $76(81.7)$ \\
Indistinct & $17(18.3)$ \\
Geographic & 0 \\
Mottled & \\
US distribution $(\mathrm{n}=93)$ & $89(95.7)$ \\
Focal & $4(4.3)$ \\
Regional & \\
Power Doppler signal $(\mathrm{n}=79)$ & $57(72.2)$ \\
Absent & $22(27.8)$ \\
Present &
\end{tabular}

Note - Data are the number of lesions.

tological characteristics of the pathologically confirmed lesions. The fibrocystic change was the most common benign histology (fig 3). Two cases of malignant lesions were a case of DCIS (fig 4) and a case of intraductal papillary carcinoma (fig 5). The patient with DCIS had a past history of breast cancer surgery on her contralateral breast and the lesion was detected as a new one during postoperative screening US. The other patient was an asymptomatic patient with average risk factors.

In US image classification, the most common type was an indistinct pattern $(81.7 \%, 76$ of 93$)$, a focal distribution $(95.7 \%, 89$ of 93$)$, absent vascularity $(72.2 \%, 57$ of 79) (Table II). Both malignant lesions showed indistinct patterns, focal distributions and presence of internal vascularity.

\section{Discussions}

Some clinical trials and observational studies have evidenced that supplemental screening breast US in conjunction with mammography have increased the cancer detection yield by $2.02-4.2$ cancers per 1000 women over that of mammography alone. It is beneficial for detecting small size cancers when they are at an earlier stage rather than they would have been if they had been detected later as interval cancers $[14,15]$. However, the detection of additional cancers is associated with a substantial callback rate as well as an increased false positive biopsy rate [16]. Several studies show low PPV and do not meet the $25-40 \%$ PPV of biopsy recommended by the US agency for Health care Policy and Research [14]. Advances in US image quality and increasing utility of bilateral whole breast US have allowed the detection of a hypoechoic area that does not meet the criteria of a mass in clinical settings and they include various spectrum, such as duct changes, architectural distortion, and non-mass lesions. However, as the current ACR BIRADS lexicon only covers mass lesions and a standard- ized method for categorization of a NML does not exist, radiologists may recommend a follow-up or biopsy for these lesions based only on their experience. As a result, it may affect the sensitivity, specificity, recall rate and PPV of screening breast US.

There have been a few studies regarding NMLs on breast US [3-6,13] and all of these studies had different inclusion criteria. Morishima et al [13] reported the sonographic characteristics of breast cancer that showed NMLs. Kim et al [3] focused only on a NML showing a nonductal hypoechoic area which was similar to that in our study and Ko et al [6] included various spectrum of NMLs including a non-ductal pattern, duct changes, architectural distortion, and posterior shadowing type. Ko et al [5] reported the potential role of shear-wave elastography for the analysis of pathologically confirmed NMLs. All of these studies included both screening and diagnostic US examinations. In two studies that compared imaging and clinical features of benign and malignant NMLs, Kim et al [3] reported that malignant NMLs more frequently had densities and calcifications on mammography and palpability on physical examination ( $p=.0052, p<0.0001, p<0.0001$, respectively). Ko et al [6] reported that NMLs with mammographic findings of suspected malignancy or presence of abnormal axillary nodes had a higher probability of malignancy $(\mathrm{p}<0.001$ and $\mathrm{p}<0.03$, respectively). These results mean that a malignant NML is significantly associated with the presence of mammographic suspicious features of malignancy or clinical symptoms. In other words, the predominant factors which affect the management of sonographic NMLs such as biopsy or follow-up are not US features but mammographic or clinical findings. Therefore, it is necessary to investigate the histologic characteristics and the likelihood of malignancy associated with these features of NMLs on screening breast US in patients with no mammographic features suspicious for malignancy.

In our study population, the incidence of NMLs was $1 \%$ and different from the incidences reported in two previous studies, which were $5.3 \%$ and $0.28 \%$, respectively. The variability in the incidence rate is thought to be due to a difference in inclusion criteria among studies. The likelihood of malignancy was $2.2 \%$ (2 of 93) and it may be classified as a BI-RADS category $4 \mathrm{a}$ lesion and tissue diagnosis is warranted, although this study had a small population of patients. It is not possible to compare the likelihood of malignancy with that in previous studies, 9.2\% (30 of 156) in the study by Kim et al [3], 27\% (38 of 164) in the study by Ko et al [6], and 35\% (12 of 34) in the study by Ko et al [5], because the present study which included only screening patients with negative findings on mammography had a different study popula- 
tion. The PPV of biopsy for NMLs in this study was $6.3 \%$ and it was similar to the PPV range of screening breast ultrasound that have been reported over the recent years, which ranged from $6.5 \%$ to $10.5 \%$ [17-21].

In the present study the management of NMLs was determined by operator experience and clinical judgement because there is no standardized guideline for the interpretation of NMLs. Although we recommended tissue diagnosis for NMLs according to our own criteria, the US features of two malignant lesions did not match up the criteria for biopsy recommendation. In other words, we recommended biopsy in cases of NMLs showing more hypoechoic patterns or posterior shadowing. However, all malignant lesions evidenced a mild hypoechoic pattern and no posterior features. In one of the malignant cases, the reason for biopsy recommendation was that the NML was a new lesion during postoperative screening US in a high risk patient with a history of contralateral breast cancer surgery. The other malignancy was initially assessed as category 3 and follow-up US after 6 months was recommended. But, biopsy was performed due to the patient's request. Therefore, further investigation will be required to establish the management guidelines for NMLs.

Fibrocystic change was the most common benign pathology $(26.7 \%, 8$ of 30$)$ and this finding was similar to the results of the two previous studies $[3,6]$. Two malignant lesions were intraductal carcinomas which is not an invasive carcinoma. DCIS lesions have been reported to be identified as NMLs of ductal or non-ductal pattern with or without echogenic spots corresponding to the mammographic findings $[7,8]$ and Shin et al [9] reported that 17 of 106 DCIS lesions (16\%) were shown as NML on screening detected DCIS. ILC also may not form a mass because it is composed of non-cohesive cells individually dispersed or arranged in a single-file linear pattern within fibrous stroma $[4,10]$. In Selinko et al series [12], 15\% of ILC were described as an ill-defined area of altered, hypoechoic, inhomogeneous echotexture without identifiable margins and without frank shadowing. Therefore, careful categorization and management of NMLs are needed in spite of the low likelihood of malignancy.

Although we analyzed the US features of NMLs, we could not compare between the benign and malignant groups statistically because of the small sample size. The most common group was indistinct pattern (81.7\%), which is consistent with that of a previous study [3]. Two malignant cases showed internal vascularity in power Doppler images. There was overlap in the Doppler signals between benign and malignant lesions, but Doppler can detect neovascularization which is an important role in the growth and extension of malignant neoplasm [22]. Furthermore, several studies reported that shear-wave elastographic features may be helpful for increasing the PPV of biopsy and reducing the number of unnecessary benign biopsies for NMLs $[5,23]$ although we could not utilize shear-wave elastography to characterize them. Therefore, biopsy burden in NMLs could be amended by the complementary use of Doppler US image or shearwave elastography. Also the use of the breast MRI might also be helpful.

This study had some limitations. Firstly, we had a small sample population with 93 NMLs including only two malignant lesions and it was not possible to perform a comparative analysis between the benign and malignant groups. Secondly, this study was retrospective and included the opportunistic screening population in one institution and there may have been a selection bias. Thirdly, the determination of NMLs on US has inherent subjectivity and can be highly operator dependent. Finally, we did not utilize shear-wave elastography to characterize the breast lesions.

\section{Conclusion}

The incidence of non-mass lesions which is recognized during screening breast ultrasound was $1.0 \%$ and the likelihood of malignancy was greater than $2 \%$. It could be classified as a BI-RADS category $4 \mathrm{a}$ lesion and tissue diagnosis is warranted. However, further prospective studies in a large, multicenter screening population are required to establish which management recommendation is most appropriate for the NMLs on screening breast US.

Acknowledgments: This work was supported by the Dong-A University Research Fund.

\section{Conflict of interest: none}

\section{References}

1. Mendelson EB, Bohm-Velez Marcela, Berg WA, et al. American College of Radiology. Breast Imaging and Reporting and Data System (ACR BI-RADS ${ }^{\circledR}$ Atlas), 5th ed. Reston, VA: American College of Radiology; 2013.

2. Japanese Association of Breast and Thyroid Sonology. Guideline for Breast Ultrasound: Management and Diagnosis. Tokyo, Japan: Nankodo Co; 2004.

3. Kim SJ, Park YM, Jung HK. Nonmasslike lesions on breast sonography. J Ultrasound Med 2014;33:421-430.

4. Uematsu T. Non-mass-like lesions on breast ultrasonography: a systematic review. Breast Cancer 2012;19:295-301. 
5. Ko KH, Jung HK, Kim SJ, Kim H, Yoon JH. Potential role of shear-wave ultrasound elastography for the differential diagnosis of breast non-mass lesions: preliminary report. Eur Radiol 2014;24:305-311.

6. Ko KH, Hsu HH, Yu JC, et al. Non-mass-like breast lesions at ultrasonography: Feature analysis and BI-RADS assessment. Eur J Radiol 2015;84:77-85.

7. Moon WK, Myung JS, Lee YJ, Park IA, Noh DY, Im JG. US of Ductal carcinoma in situ. Radiographics 2002;22:269-280.

8. Wang LC, Sullivan M, Du H, Feldman MI, Mendelson EB. US appearance of ductal carcinoma in situ. Radiographics 2013;33:213-228.

9. Shin HJ, Kim HH, Kim SM, Kwon GY, Gong G, Cho OK. Screening-detected and symptomatic ductal carcinoma in situ: differences in the sonographic and pathologic features. AJR Am J Roentgenol 2008;190:516-525.

10. Lopez JK, Bassett LW. Invasive lobular carcinoma of the breast: spectrum of mammographic, US, and MR imaging findings. Radiographics 2009;29:165-176.

11. Butler RS, Venta LA, Wiley EL, Ellis RL, Dempsey PJ, Rubin E. Sonographic evaluation of infiltrating lobular carcinoma. AJR Am J Roentgenol 1999;172:325-330.

12. Selinko VL, Middleton LP, Dempsey PJ. Role of sonography in diagnosing and staging invasive lobular carcinoma. J Clin Ultrasound 2004;32:323-332.

13. Morishima I, Ueno E, Tohno E, Tsunoda-Shimizu H, Kujiraoka Y, Takasaki M. Ultrasonic diagnosis of non-mass image-forming breast cancer. In: Ueno E, Shiina T, Kubota M, Sawai K (eds). Research and Development in Breast Ultrasound. Tokyo, Japan: Springer; 2005:127-134.

14. Brem RF, Lenihan MJ, Lieberman J, Torrente J. Screening breast ultrasound: past, present, and future. AJR Am J Roentgenol 2015;204:234-240.

15. Ohuchi N, Suzuki A, Sobue T, et al. Sensitivity and specificity of mammography and adjunctive ultrasonography to screen for breast cancer in the Japan Strategic Anti-cancer Randomized Trial (J-START): a randomised controlled trial. Lancet 2016;387:341-348.

16. Kelly KM, Dean J, Comulada WS, Lee SJ. Breast cancer detection using automated whole breast ultrasound and mammography in radiographically dense breasts. Eur Radiol 2010;20:734-742.

17. Berg WA, Blume JD, Cormack JB, et al. Combined screening with ultrasound and mammography vs mammography alone in women at elevated risk of breast cancer. JAMA 2008;299:2151-2163.

18. Kolb TM, Lichy J, Newhouse JH. Comparison of the performance of screening mammography, physical examination, and breast US and evaluation of factors that influence them: an analysis of 27,825 patient evaluations. Radiology 2002;225:165-175.

19. Kaplan SS. Clinical utility of bilateral whole-breast US in the evaluation of women with dense breast tissue. Radiology 2001;221:641-649.

20. Weigert J, Steenbergen S. The Connecticut experiment: the role of ultrasound in the screening of women with dense breasts. Breast J 2012;18:517-522.

21. Hooley RJ, Greenberg KL, Stackhouse RM, Geisel JL, Butler RS, Philpotts LE. Screening US in patients with mammographically dense breasts: initial experience with Connecticut Public Act 09-41. Radiology 2012;265:59-69.

22. Del Cura JL, Elizagaray E, Zabala R, Legorburu A, Grande D. The use of unenhanced Doppler sonography in the evaluation of solid breast lesions. AJR Am J Roentgenol 2005;184:1788-1794.

23. Choi JS, Han BK, Ko EY, Ko ES, Shin JH, Kim GR. Additional diagnostic value of shear-wave Elastography and color Doppler US for evaluation of breast non-mass lesions detected at B-mode US. Eur Radiol 2016;26:35423549 . 